\section{MS21-P9 Relation between \\ photoluminescence properties and cristalline structure of III-V semiconductor alloys grown by MOVPE \\ Tatiana Prutskij ${ }^{1}$, Nykolay Makarov $^{1}$, Giovanni Attolini ${ }^{2}$}

1. Instituto de Ciencias, Benemerita Universidad Autonoma de Puebla, Puebla, Mexico

2. Istituto dei Materiali per l' Elettronica ed il Magnetismo, CNR, Parma, Italy

\section{email: tatiana.prutskij@correo.buap.mx}

III-V semiconductor alloys, such as a ternary GaInP and a quaternary GaInAsP alloys, are important materials widely used in semiconductor lasers, solar cells, high electron mobility transistor (HEMT) and heterojunction bipolar transistors (HBT). It is well known that within III-V semiconductor epitaxial layers grown by Metalorganic Vapour Phase Epitaxy (MOVPE) technique a periodic composition fluctuation coexists with spontaneously-formed atomically-ordered clusters. The photoluminescence (PL) emission characteristics strongly depend on the crystalline structure. In particular, the PL emission of epitaxial III-V layers with atomic ordering is polarized and the polarization degree depends on the values of ordering parameter and elastic, biaxial strain.

We studied the PL emission polarization of the GaInP and GaInAsP epitaxial layers lattice matched to GaAs. We measured and calculated the integrated PL intensity as a function of polarization angle for the PL emission of two atomically ordered III-V alloys with different ordering parameters. We compared these measured and calculated PL polarization patterns for the PL propagating along the [001] direction, and along [1-10] and [110] directions. Our calculations show that if the alloy is almost lattice-matched to the substrate the PL emission from the layer (001) surface is polarized because of atomic ordering in the alloy, and the PL polarization degree is proportional to the ordering parameter. The polarization degree of the PL emission from the (001) surface reaches its maximum when the lattice parameters of the layer and the substrate match, and decreases proportionally to the value of biaxial strain. The PL from the edge planes is strongly polarized when the epitaxial layer is under a strong biaxial strain, thus the strong strain becomes a dominant factor in the polarization degree. We measured the polarization of the PL emission from the (001) surface the ordered GaInP and GaInAsP alloy at different temperatures and analyzed its temperature evolution within the range from $10 \mathrm{~K}$ to $300 \mathrm{~K}$. We observed the change of the PL polarization pattern induced by thermal strain due to the difference between the thermal expansion coefficients of the layer and the substrate, and estimated the biaxial strain at different temperatures. Our measurements point out that the polarization of the PL emission from (001) plane increases with decreasing temperature because of the thermal variation of the epitaxial strain.

Keywords: III-V solid solutions, MOVPE, Photoluminecscence

\section{MS21-P10 Crystallographic Investigation of Photo-Excited States in Molecular Crystals \\ Nikolaj Roth ${ }^{1}$}

1. Center for materials crystallography, Department of chemistry, Aarhus university, Denmark

email: nikolajroth@chem.au.dk

Materials with photo-switchable physical properties become increasingly studied for possible applications. These controllable properties, such as changes in magnetic susceptibility ${ }^{1}$ and electric polarisation ${ }^{2}$, often come from electronically excited states of the constituent molecules of the crystals. The study of electronically excited states in molecules has traditionally been carried out using spectroscopic techniques, giving very accurate values for energy levels, but only rough estimates of the structural changes associated with the excitation. The structural investigation of these states can instead be carried out using the methods of photo-crystallography, a field which is currently seeing rapid development. By exciting a crystal with light, the resulting structural changes can be probed using $\mathrm{x}$-ray and neutron diffraction techniques. Here, a long lived excited state of the photo-magnetic compound $\left[\mathrm{Nd}(\mathrm{DMF})_{4}\left(\mathrm{H}_{2} \mathrm{O}\right)_{3}(\mu-\mathrm{CN}) \mathrm{Fe}(\mathrm{CN})_{5}\right] \cdot \mathrm{H}_{2} \mathrm{O}$ is investigated using single-crystal $\mathrm{x}$-ray diffraction. The excitation is carried out using a new LED-based device which can easily be mounted on most diffractometers and present a cheap alternative to laser-based setups ${ }^{3}$. It is found that the excited state is more stable than previously thought ${ }^{4}$, as it can be kept for hours at 100K. [1] Gütlich, P et al. (2001) Coord. Chem. Rev. 219-221, 839-879 [2] Collet et al. (2003) SCIENCE, 300, 612-615 [3] Brayshaw et al. (2010) J. Appl. Cryst. 43, 337-340 [4] Svendsen et al. (2009) Angew. Chem. 121, 2818-2821

Keywords: photocrystallography, excited state, photo-induced 